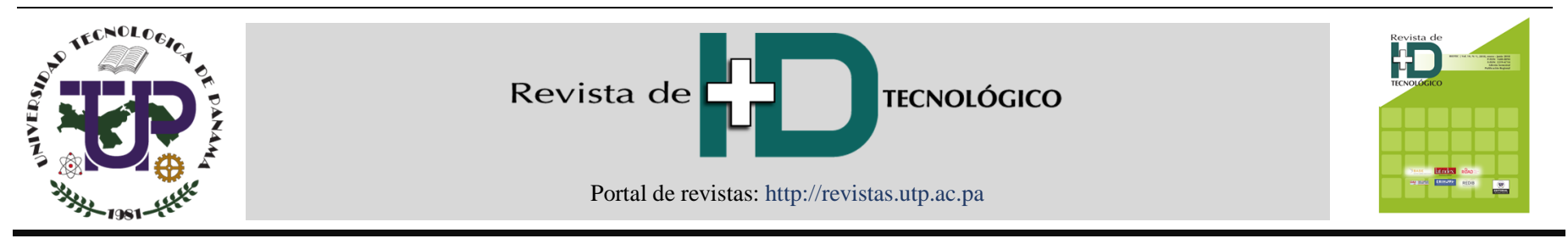

\title{
Las Tecnologías de información y comunicación un gran reto para las PYMES del distrito de Changuinola, República de Panamá
}

\section{ICT a big challenge for SMEs in the district of Changuinola, Republic of Panama}

\author{
José Mendoza Avilés ${ }^{1 *}$ \\ ${ }^{1}$ Centro Regional de Bocas del Toro Universidad Tecnológica de Panamá, Panamá \\ *Autor de correspondencia: jose.mendoza@utp.ac.pa
}

\begin{abstract}
RESUMEN- En este trabajo se analiza el nivel Académico-Técnico del Personal Administrativo y su relación con la aplicación de las Tecnologías de la Información y Comunicación en las Pequeñas y Medianas Empresas del distrito de Changuinola. Se aplicó un diseño no experimental, tipo descriptiva y transversal, en la cual participaron 114 PYMES del distrito de Changuinola, provincia de Bocas del Toro, República de Panamá que, para garantizar la presencia de los subgrupos de todos los corregimientos del distrito dentro de la muestra, se trabajó con el muestreo estratificado por conglomerado. Se utilizó la técnica de encuesta con el instrumento cuestionario. Los resultados indican que, existe relación entre nivel académico o capacitación técnica de los propietarios o/y administradores de las PYMES con la frecuencia de uso de las TIC en la empresa. Por lo tanto, se concluyó que, un alto nivel académico o capacitación técnica del personal administrativo y adecuado equipamiento en hardware, software y conectividad en las PYMES beneficia el uso eficiente de las tecnologías de la información y la comunicación.
\end{abstract}

Palabras clave- PYMES, TIC, Nivel Académico, Equipamiento TIC.

ABSTRACT - This paper analyzes the Academic-Technical level of the Administrative Staff and its relationship with the Application of Information and Communication Technologies in Small and Medium Enterprises in the district of Changuinola. A non-experimental, descriptive and cross-sectional design was applied, in which 114 PYMES from the district of Changuinola, province of Bocas del Toro, Republic of Panama participated, to guarantee the presence of the subgroups of all the subdivisions of the district within the sample, we worked with stratified sampling by conglomerate. The survey technique was used with the questionnaire instrument. The results indicate that there is a relationship between academic level or technical training of owners or/and administrators of SMEs with the frequency of use of ICT in the company. Therefore, it was concluded that a high academic level or technical training of administrative personnel and adequate equipment in hardware, software and connectivity in SMEs benefits the efficient use of information and communication technologies.

Keywords-SME, ITC, Academic level, ICT equipment.

\section{Introducción}

La incorporación de las tecnologías de información y comunicación (TIC), beneficia a las empresas en la medida que posibilita la reducción de los costos de transacción y el incremento de la eficiencia de los procesos.

Según [1], un desafío clave dentro de la industria de servicios es mejorar la percepción de cómo los gerentes diferencian los beneficios de la adopción y difusión de TIC y cómo se relacionan realmente con el nivel de madurez con estas tecnologías. Las TIC influyen en la productividad cuando los niveles más altos de inversión financiera en TIC generan nuevos productos y baja de los precios [2].

El crecimiento de las pequeñas empresas es tanto horizontal como vertical. El crecimiento horizontal está representado por nuevas microempresas que se gradúan en pequeñas empresas, mientras que el crecimiento vertical ocurre cuando las pequeñas empresas se gradúan en empresas medianas. Este crecimiento está determinado por las decisiones tomadas por los empresarios sobre los cambios que tienen lugar tanto en el entorno empresarial externo como en el interno. Uno de esos cambios ha sido la rápida revolución de la Tecnología de la Comunicación de la Información que posee oportunidades y amenazas para las pequeñas empresas [3].

Cabe resaltar el estudio de la UIT [4], "El extraordinario auge de las tecnologías de la información y la comunicación incide, considerablemente, en el crecimiento económico de los países tanto desarrollados como en desarrollo. Los sectores productores de TIC (prestatarios de servicios y fabricantes) generan beneficios directos e indirectos en los países donde están

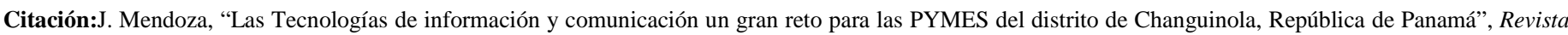
de I+D Tecnológico, vol. 14, no. 2, pp. (49-56), 2018.

Tipo de artículo: Original. Recibido: 28 de febrero de 2018. Recibido con correcciones: 30 de noviembre de 2018 Aceptado: 30 de noviembre de 2018 . Copyright: $2018 \mathrm{~J}$. Mendoza. This is an open access article under the CC BY-NC-SA 4.0 license (https://creativecommons.org/licenses/by-nc-sa/4.0/). 
establecidos. El crecimiento de estas industrias redunda, directamente, en la creación de empleo y en mayores ingresos. La magnitud de dichos beneficios directos depende de la proporción que representan los sectores productores de bienes y servicios relativos a las TIC en la economía y de su ritmo de crecimiento". El uso de los servicios de las TIC, como los teléfonos móviles e Internet, continúa creciendo en todo el mundo, como se muestra en el estudio realizado por ITU, como se puede observar en la figura 1 .

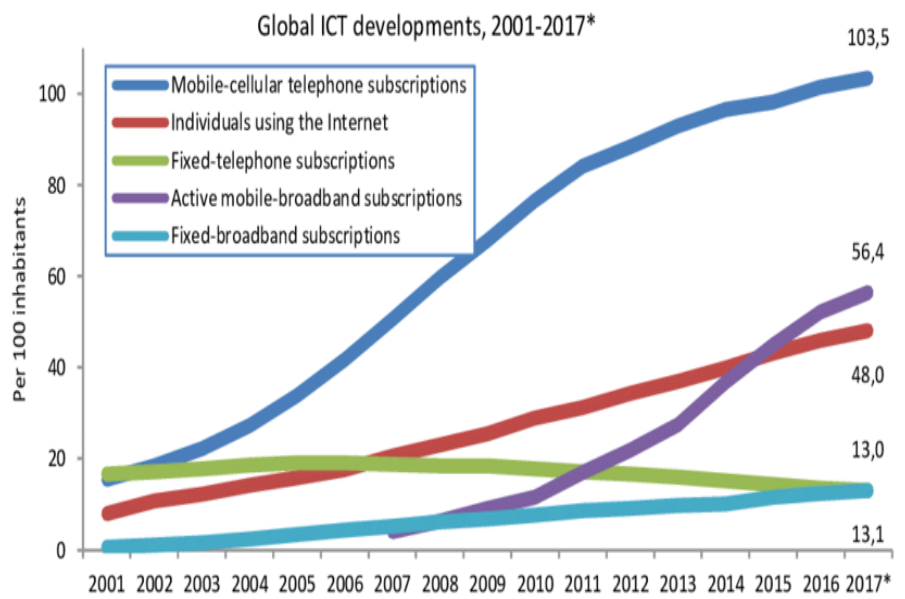

Figura 1. Desarrollo global de las TIC del 2001-2017. Fuente: [5].

Según el estudio realizado por la Comisión Económica para América Latina y el Caribe (CEPAL) de las Naciones Unidas, sobre las políticas públicas y desarrollo productivo en América Latina, [6], se puede notar que las distancias con la Organización para la Cooperación y el Desarrollo Económico (OCDE) se han reducido en 'infraestructura y acceso', mientras que el mayor aumento de brecha se verifica en 'uso de TIC'. Esto refleja el enfoque dominante de las estrategias implementadas en los países de la región, que se han concentrado, esencialmente, en programas orientados a mejorar la infraestructura y el acceso. Al mismo tiempo, es importante resaltar que existe una importante heterogeneidad al interior de la región, donde se observa que los países del Cono Sur y del Caribe se encuentran mejor posicionados, mientras que los de América Central muestran un mayor atraso.

En la parte superior de la clasificación se encuentran los dos grandes países desarrollados de la región, los Estados Unidos y Canadá. Ambos se ubican dentro de los 30 países más importantes del mundo, pero están por detrás de los países desarrollados en Europa y Asia, que tienen un Ingreso Nacional Bruto (INB) per cápita comparablemente alto, principalmente debido a los valores más bajos del ancho de banda de Internet internacional por usuario de Internet, computadora doméstica y acceso a Internet. Panamá se encuentra por encima del promedio de los países en desarrollo, pero por debajo del promedio mundial y el continente americano, y muy por debajo de Estados Unidos y Canadá pertenecientes a los países desarrollados (ver figura 2).

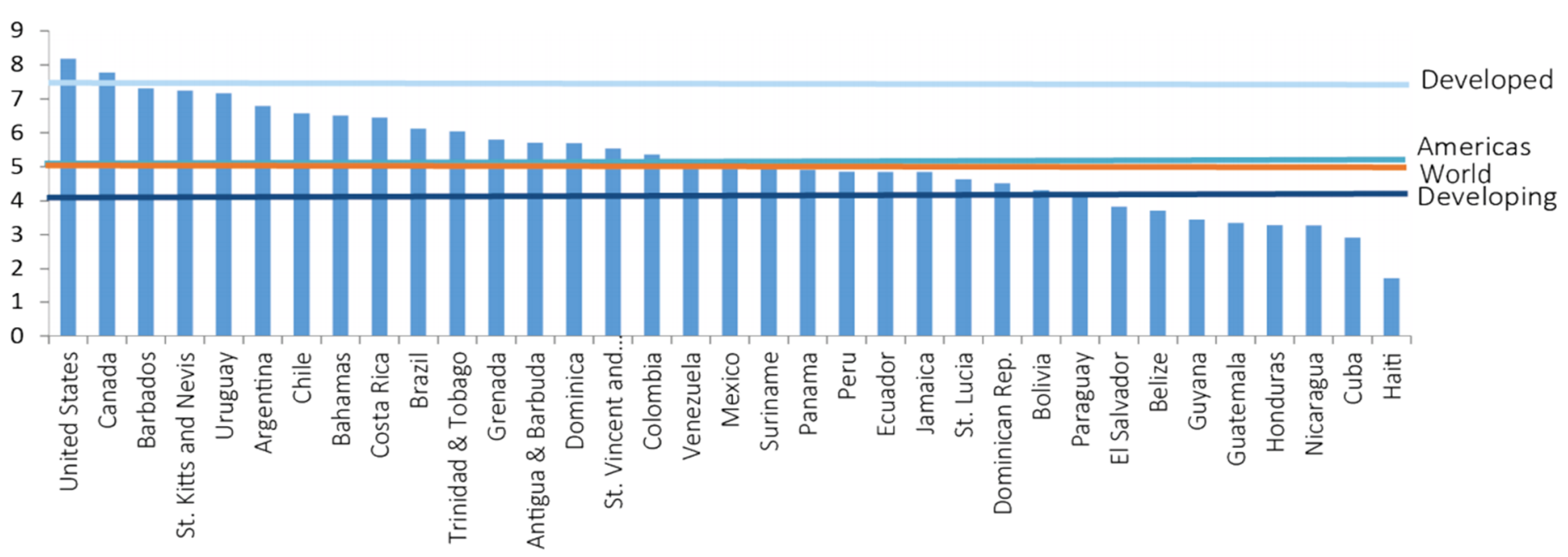

Figura 2. Índice de desarrollo de las TIC en el continente americano al 2017. Fuente: [5]. 
Los valores del Índice de Desarrollo de las TIC (IDT) durante el año también cayó por debajo del promedio mundial. Otros tres países de la región se encuentran dentro del cuartil superior de las clasificaciones mundiales: los Estados insulares del Caribe de Barbados, San Cristóbal y Nieves, y Uruguay en Sudamérica, que ha subido seis puestos en el ranking mundial, del 48 al 42 , después de mejorar su valor de IDI en 0.41 puntos [5].

En las empresas y países que asimilan nuevas tecnologías e innovaciones, se esperan cambios rápidos. Existen diferentes estudios que analizan las TIC, especialmente los factores que afectan la adopción de TIC. El análisis de los efectos de las tecnologías en el sector privado solo es significativo después de un análisis exhaustivo de las condiciones que deben satisfacerse para adoptar las TIC: expectativas de impactos positivos de estas tecnologías y las características de cada empresa están estrechamente relacionados, como recursos financieros, tecnológicos, de personal, flexibilidad de las estructuras, etc. [7].

El último censo formal del Instituto Nacional de Estadísticas y Censo de la Contraloría General de la República de Panamá refleja la cantidad de empresas, en el territorio nacional, distribución que se observa en la tabla 1.

Tabla 1. Número de empresas en la República, por naturaleza jurídica, según provincia: año 2009. Fuente: [8]

\begin{tabular}{l|r|r|r|r}
\hline \multirow{2}{*}{ Provincia } & \multicolumn{4}{|c}{ Número de Empresas } \\
\cline { 2 - 5 } & \multirow{2}{*}{ Total } & \multicolumn{3}{|c}{ Naturaleza Jurídica } \\
\cline { 3 - 5 } & & Individual & Anónima & \multicolumn{1}{c}{ Otras } \\
\hline TOTAL & $\mathbf{6 0 , 4 4 4}$ & $\mathbf{4 2 , 9 1 0}$ & $\mathbf{1 5 , 5 3 4}$ & $\mathbf{2 , 0 0 0}$ \\
\hline Bocas del Toro & 1,483 & 1,344 & 126 & 13 \\
Coclé & 3,864 & 3,410 & 374 & 80 \\
Colón & 3,871 & 2,443 & 1,331 & 97 \\
Chiriquí & 7,861 & 6,548 & 1,161 & 152 \\
Darién & 962 & 935 & 15 & 12 \\
Herrera & 3,084 & 2,556 & 334 & 194 \\
Los Santos & 2,723 & 2,310 & 205 & 208 \\
Panamá & 32,334 & 19,523 & 11,655 & 1,156 \\
Veraguas & 4,262 & 3,841 & 333 & 88 \\
\hline
\end{tabular}

Según la Ley 8 del año 2000 [9], por la cual se crea la Autoridad de la Micro, Pequeña y Mediana Empresa, denominada AMPYME, en el artículo 5 define la micro, pequeña y mediana empresa:

- Microempresa: Unidad económica, formal o informal, que genere ingresos brutos o facturación anuales hasta la suma de ciento cincuenta mil balboas (B/. 150,000.00).

- Pequeña empresa: Unidad económica que genere ingresos brutos o facturación anuales desde ciento cincuenta mil balboas con un centésimo (B/. 150,000.01) hasta un millón de balboas (B/. $1,000,000.00)$.

- Mediana empresa: Unidad económica que genere ingresos brutos o facturación anuales desde un millón de balboas con un centésimo (B/. 1,000,000.01) hasta dos millones quinientos mil balboas (B/. 2,500,000.00).

Actualmente, existen registradas en la AMPYME más de 60.000 pequeñas y medianas empresas, las cuales le brindan sustento a más de medio millón de personas en todo el país, según [10].

Uno de los más importantes desarrollos tecnológicos de los últimos años ha sido el de las tecnologías de la información y las comunicaciones. Los beneficios potenciales de estas tecnologías para una organización incluyen desde los beneficios de carácter operativo, como el aumento de la eficiencia, [11].

"Las micro, pequeñas y medianas empresas (MIPYMES) representan el principal motor de la economía en América Latina con proyecciones de crecimiento favorables para los próximos años y con una tendencia a la adopción de herramientas tecnológicas que faciliten las labores al interior de sus organizaciones, es así que de acuerdo con un estudio de Brother International Corporation realizado con pequeñas y medianas empresas (PYMES), el 49\% de estas afirmó que la inversión en elementos tecnológicos sería su prioridad para el presente año", [12].

El estudio realizado por [13], presenta que, las Tecnologías de Información y Comunicación están logrando incrementar el desarrollo eficaz y productivo en las PYME. Su utilización y aplicación aporta una serie de ventajas potenciales en todas las áreas funcionales de la empresa, permitiendo una mayor agilidad en la generación, acceso y distribución de la información, una mayor coordinación en la toma de decisiones, una mejora en la conexión y asistencia a clientes y proveedores. Desde diversos ámbitos se afirme que la correcta utilización de las TIC en la organización de la PYME puede resultar una importante ventaja competitiva.

Uno de los ítems de gran relevancia en la implementación de las TIC son las infraestructuras, se entienden por el conjunto de medios físicos (hardware), 
la parte blanda de las tecnologías (software) y las redes de comunicación, que incluyen líneas y equipos de transmisión, así como nodos de conmutación y los equipos servidores, que albergan los contenidos e informaciones a los que acceden los usuarios.

Uno de los factores que influyen en la decisión de adopción, así como los beneficios operativos y estratégicos que brinda el uso de TIC, es diferenciarse de la competencia; teniendo presente los cambios que requiere una organización para brindar un adecuado uso a las TIC adoptadas, lo cual se relaciona con el tamaño de la empresa, su actividad económica, su experiencia previa con las TIC, la escasez relativa de recursos y la centralización de la toma de decisiones [14].

De acuerdo con los temas analizados, en la figura 3 se propone un modelo conceptual que resume las variables relacionadas con las TIC y que tienen en principio un impacto en la competitividad de las PYMES. Las principales variables de las TIC son las competencias (dominio y conocimientos), sus capacidades (Hardware, Software, Internet, Personal), su potencial de Inversión, el alineamiento con la estrategia del negocio, y la generación de ventajas competitivas para la organización [16].

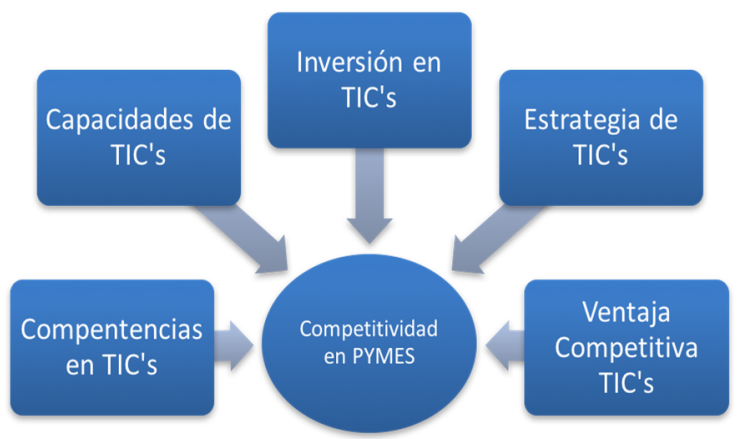

Figura 3. Modelo de Competitividad de las PYMES y las TIC. Fuente: [15].

Según la investigación [17], en Panamá el tema tecnológico se encuentra en el último lugar (17) entre las prioridades de las PYMES. Sin embargo, los hallazgos de la investigación indican que:

- Un porcentaje significativo de estas empresas emplea tecnología tradicional;

- quienes invierten en tecnología son una proporción mínima;

- los altos costos de la tecnología son una de las razones de esta deficitaria inversión;
- no hay clara conciencia de los beneficios de la adopción de nueva tecnología;

- y este fenómeno es más recurrente en las pequeñas empresas que en las medianas.

Un estudio realizado por la Fundación para el Desarrollo Sostenible en América Latina [18], presenta en su avance de investigación, los obstáculos al desarrollo de las pequeñas y medianas empresas en América Latina de un trabajo que se realiza en seis países: Argentina, Colombia, Costa Rica, Chile, El Salvador y Panamá, donde resume que: uno de los aspectos relevante guarda relación con la calidad del recurso humano. En muchos casos, se hace referencia a la poca calificación (teórica y práctica) del personal. Esto tiene que ver, por un lado, con la formación básica y, eventualmente, técnica $\mathrm{y}$, por otro lado, con la experiencia previa. Claramente, se observa que existe un divorcio entre la formación y las necesidades del mercado laboral, razón por la cual es clave en este aspecto el papel del sistema educativo y de las propias empresas.

Según [19], la educación continua podría ser una opción para llevar actualización en el área de las tecnologías de información y comunicación. Una de las grandes características que posicionan la educación continua son los cursos de capacitación, talleres, seminarios, workshop y diplomados, ya que son más específicos y responden de manera directa y rápida a la demanda de las instituciones, organizaciones y colaboradores de grandes compañías.

La capacitación se ha vuelto una de las prioridades en el sector de Pequeñas y Medianas Empresas en Panamá, en donde los pequeños empresarios han tomado conciencia de que sin los conocimientos adecuados no podrán mantener sus negocios a largo plazo, [10].

En base a los antecedentes recabados se pretende dar respuesta a la pregunta de investigación, ¿Cuál es el nivel Académico-Técnico del Personal Administrativo y su relación con la Aplicación de las Tecnologías de la Información y Comunicación en las Pequeñas y Medianas Empresas del distrito de Changuinola?

Esta investigación es de vital importancia para los empresarios, la cámara de comercio, industrial y agropecuaria de Bocas del Toro (CCIAB) y la Autoridad de la Micro, Pequeña y Mediana Empresa (AMPYME) del distrito de Changuinola, provee datos sobre la incorporación de las TIC en las PYMES en el distrito de Changuinola, las cuales generan un alto porcentaje de 
empleo y de valor agregado, por lo que se consideran actores centrales en este trabajo.

\section{Materiales y métodos}

Este estudio se desarrolló con las Pequeñas y medianas empresas del distrito de Changuinola, provincia de Bocas del Toro, Panamá, región noroccidental fronteriza con Costa Rica en donde funcionan, actualmente, 162 PYMES, con ingresos que van desde los 70,000.00 hasta los 2,500,000.00 de dólares, saliendo una muestra de 114 con un nivel confianza del $95 \%$ y un margen de error de $5 \%$. Para garantizar presencia de pequeñas y medianas empresas de todos los corregimientos del distrito de Changuinola, se procedió a realizar un muestreo estratificado por conglomerado, como se muestra en la tabla 2.

Tabla 2. Sujetos y Muestra de los estratos

\begin{tabular}{llrrr}
\hline Estrato & Identificación & Sujetos & Proporción & Muestra \\
\hline 1 & Centro & 64 & $39,5 \%$ & 45 \\
2 & Cuadrante & 6 & $3,7 \%$ & 4 \\
3 & El Empalme & 31 & $19,1 \%$ & 22 \\
4 & Finca 11 & 15 & $9,3 \%$ & 11 \\
5 & Finca 4 & 6 & $3,7 \%$ & 4 \\
6 & Finca 6 & 9 & $5,6 \%$ & 6 \\
7 & Finca 8 & 5 & $3,1 \%$ & 4 \\
8 & Guabito & 17 & $10,5 \%$ & 12 \\
9 & Las 60 y 30 & 9 & $5,6 \%$ & 6 \\
\hline & & $\mathbf{1 6 2}$ & $\mathbf{1 0 0 , 0 \%}$ & $\mathbf{1 1 4}$ \\
\hline
\end{tabular}

La unidad de análisis fueron las personas consideradas como propietarios y/o gerentes que administran las PYMES.

Esta investigación tuvo un enfoque cuantitativo porque las variables del estudio fueron medidas y cuantificadas para verificar su aplicabilidad. El diseño de la investigación fue no experimental, ya que, no se manipulan las variables del estudio. El tipo de investigación fue descriptiva, debido a que, se analizó el nivel académico y capacitación técnica del personal administrativo y su relación con el uso de las tecnologías de la información y comunicación y, Por otro lado, transversal porque se midieron las variables del estudio en un solo momento.

\subsection{Criterios}

\section{- Criterios de inclusión:}

Empresas con ingresos que van desde los 70,000.00 hasta los 2,500,000.00 de dólares.
- Criterios de exclusión:

Empresas grandes con ingresos mayores de 2,500,000.00 de dólares y Microempresas con ingresos menores de 70,000.00 dólares.

La hipótesis de investigación quedó formulada de la siguiente manera: Un alto nivel académico o capacitación técnica del personal administrativo y adecuado equipamiento en hardware, software y conectividad en las PYMES beneficia el uso eficiente de las tecnologías de la información y la comunicación.

\subsection{Instrumentos de recolección de datos}

Sobre la recolección de los datos [20], escribe que una vez seleccionamos el diseño de investigación apropiado y la muestra adecuada, de acuerdo con nuestro problema de estudio e hipótesis, se procede a recolectar los datos pertinentes sobre los atributos, conceptos o variables del estudio. Se utilizó la técnica encuesta con el instrumento cuestionario, conformado por preguntas cerradas para obtener los datos necesarios para determinar el uso de las TIC, nivel académico-técnico e infraestructura (equipamiento de hardware, software y conectividad). Para obtener esta información, se les solicitó a los administradores de las PYMES que llenaran la encuesta, divididas en tres bloques.

\section{- Variables: Nivel Académico-técnico}

El primer bloque contiene los ítems que responden al nivel académico-técnico del personal administrativo: Nivel académico, Capacitación en TIC, Cómo obtuvo el conocimiento en TIC y Experiencia en manejo de TIC.

\section{- Variable: uso de las Tecnologías de la Información y Comunicación}

Este segundo bloque contiene los ítems que responden al uso de las tecnologías de la información y comunicación entre empresas: Uso internet en el negocio, Frecuencia de uso del computador, Nivel tecnológico de la empresa, Cantidad de personas adicional que usa TIC, Uso de las tecnologías en el negocio, Uso de las TIC para comunicar la empresa, Razones porque no tiene TIC en la empresa, Recibir asesoría y/o capacitación en el manejo de TIC.

\section{- Variables: Equipamiento hardware, software y conectividad}

Este tercer y último bloque contiene los ítems que responden el tipo de equipamiento hardware, software y conectividad: Utilización de Equipamiento en TIC, Tipo de equipamiento, Conexión a Internet, Tipo de conexión 
de Internet, Tipo de comunicación Interna y Externa, y Tipo de software instalados.

\section{Resultados}

Para determinar la validez de contenido del cuestionario, se aplicó el método de agregados individuales, en donde se solicitó a tres (3) especialistas en sistemas o negocios para que certificaran si, efectivamente, las preguntas, reactivos o afirmaciones seleccionadas eran claras y coherentes con el tema investigado.

En la evaluación de confiabilidad del instrumento, se utilizó el procedimiento Test-Retest, que implica la aplicación de la misma prueba o test dos veces a los mismos sujetos. Este procedimiento nos permitió evaluar el grado de estabilidad del instrumento en el tiempo. Posteriormente, se correlacionaron los pares de puntuaciones para obtener el coeficiente de fiabilidad del método Test-Retest, el cual debe estar entre 0.7 y 1.0 para aceptar las preguntas y los que estén por debajo de 0.7 para reformularlas o eliminarlas [21]. El coeficiente de fiabilidad obtenido para instrumento en curso fue de 0.9.

Para determinar la posible relación entre el nivel académico-técnico, el equipamiento de hardware, software y conectividad con el uso de las TIC se efectuó un análisis de estadística descriptiva, correlación de Pearson y la prueba de $\mathrm{X}^{2}$ (Chi al cuadrado). Estos procedimientos se realizaron con el apoyo del programa SPSS versión 22.0 y Microsoft Excel 2016.

La figura 4 muestra, que de los propietarios y/o administradores encuestados, se destaca el hecho que, el nivel académico más notorio entre los administradores es el nivel medio (Bachillerato) con un 55.3\% y solo el $19.3 \%$ posee estudios técnicos-universitarios.

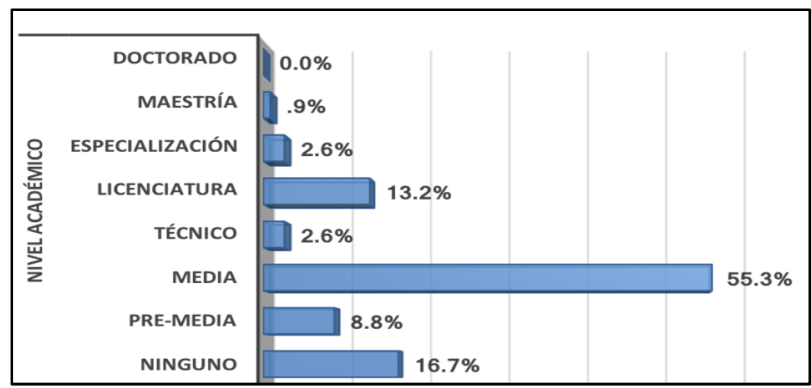

Figura 4. Nivel Académico de los administradores de las PYMES.

La figura 5 muestra que, en función del nivel de experiencia obtenida en el manejo de las TIC, el 64\% de los administradores tiene los conocimientos básicos y sólo el $23.7 \%$ tiene un nivel de experiencia en manejo de TIC de intermedia a avanzada. Por otra parte, el $34.2 \%$ no tiene conocimiento en TIC, y el 47.4 obtuvo el conocimiento en manejo de las TIC de manera empírica o por iniciativa propia. Cabe resaltar que el $77.2 \%$ restante no ha tomado capacitación en el manejo de las TIC para la empresa.

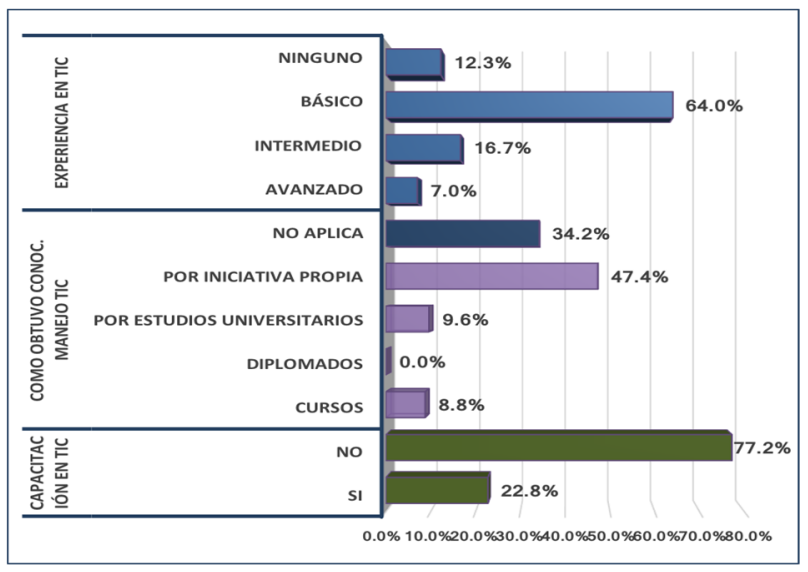

Figura 5. Capacitación, conocimiento y experiencia de los administradores de las PYMES en TIC.

La figura 6 muestra que solo el $44.7 \%$ de las empresas tienen terceras personas para manejar las TIC. De las empresas que manejan tecnología, se destaca que el $43 \%$ considera tener un débil nivel tecnológico y solo el $25.4 \%$ considera tener un fuerte nivel tecnológico.

La frecuencia con que se utiliza la computadora en el negocio, el $50 \%$ la utiliza diariamente y el $46.1 \%$ no la utiliza en el negocio.

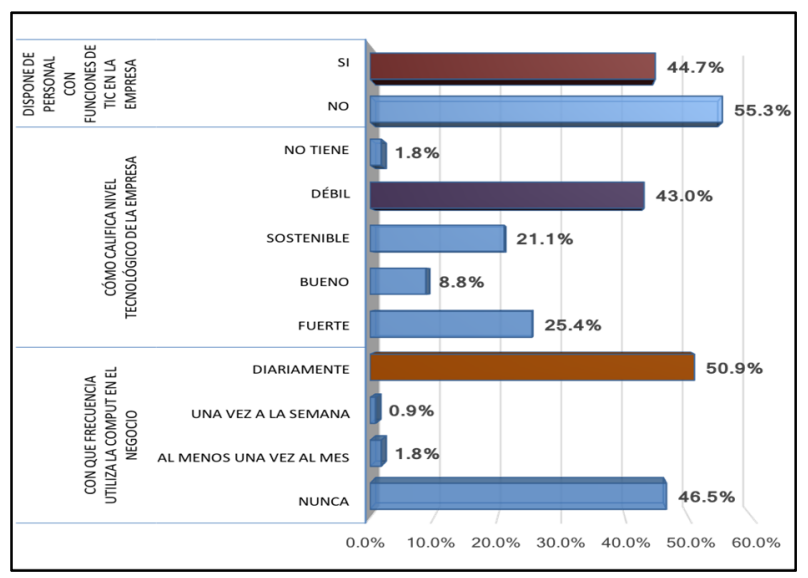

Figura 6. Personal con funciones TIC, nivel tecnológico y frecuencia de uso de las TIC en las PYMES. 
Al realizar la correlación de Pearson, encontramos que existen niveles significativos aceptables de 0.507 entre las variables nivel académico-técnico del personal administrativo y su relación con el uso de las tecnologías de la información y comunicación, las dos variables se correlacionan en sentido directo. A mayor nivel académico-técnico, mayor frecuencia de uso de las TIC y a menor nivel académico-técnico, menor frecuencia de uso de las TIC, ver figura 7.

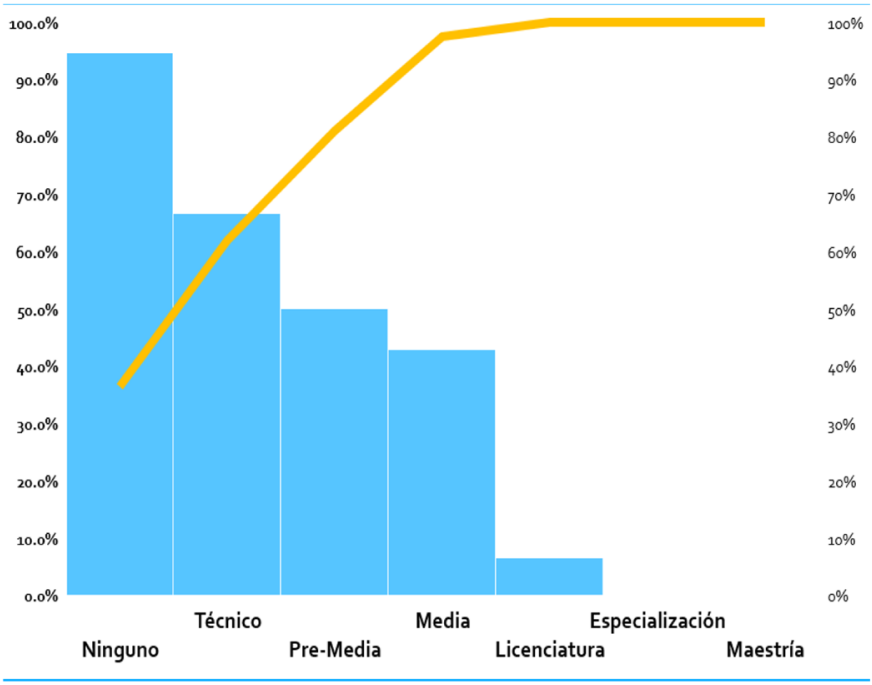

Figura 7. Relación entre el nivel académico-técnico de los administradores y la frecuencia de uso de las TIC en las PYMES.

Existe una relación entre nivel académico de los propietarios o/y administradores con la frecuencia de uso de las TIC en la empresa. Esto demuestra que a menos nivel académico menos uso frecuente de las TIC en el negocio.

$\mathrm{El} \mathrm{Chi}^{2}$ "P=0.00001" es menor que 0.01 , concluye que las dos variables nivel académico-técnico y frecuencia de uso de las TIC, no son independientes. El nivel académico-técnico está relacionado con la frecuencia de uso de las TIC y no son independientes.

El nivel académico y capacitación técnica está relacionado con la frecuencia de uso de las TIC, por ende, se demuestra, estadísticamente, que se rechaza la hipótesis nula y se acepta la hipótesis de la investigación.

\section{Conclusiones}

Al analizar el nivel académico-técnico del personal administrativo y su relación con el uso de las tecnologías de la información y comunicación en las pequeñas y medianas empresas del distrito de Changuinola podemos concluir:

Las relaciones entre nivel académico de los propietarios o/y administradores con la frecuencia de uso de las TIC en la empresa, demuestra que, a menos nivel académico, menos uso frecuente de las TIC en el negocio.

Al realizar la correlación de Pearson, se encontró que existen niveles significativos aceptables de 0.507 entre las variables nivel académico-técnico del personal administrativo y su relación con el uso de las tecnologías de la información y comunicación, las dos variables se correlacionan en sentido directo. De las PYMES encuestadas, la que poseían administradores con un mayor nivel académico-técnico tenían una frecuencia de uso mayor de las TIC en la empresa y los que tenía un menor nivel académico-técnico, tenían una frecuencia menor de uso de las TIC en el negocio.

Sobre las relaciones entre si se ha recibido capacitación en TIC y su uso frecuente por los administradores $\mathrm{y} / \mathrm{o}$ propietarios de las PYMES encuestadas, al realizar la correlación de Pearson, se encontró que existen niveles significativos aceptables entre las variables Capacitación en TIC del personal administrativo y su relación con el uso frecuente de las TIC, las dos variables se correlacionan en sentido inverso.

El $\mathrm{Chi}^{2}$ tiene una significancia de $\mathrm{p}=0.0001$ es menor que alfa, concluye que las dos variables nivel académicotécnico y frecuencia de uso de las TIC, no son independientes. Dados estos resultados se rechaza la hipótesis nula y se aprueba la hipótesis de investigación, que afirma que, un alto nivel académico o capacitación técnica del personal administrativo y adecuado equipamiento en hardware, software y conectividad en las PYMES beneficia el uso eficiente de las tecnologías de la información y la comunicación.

De este trabajo nace la idea de investigar sobre, como Internet influye en las PYMES del distrito de Changuinola.

\section{Agradecimiento}

La historia de un trabajo de investigación es, a fin de cuentas, la historia de todos los que han aportado en el desarrollo de este proyecto: de manera especial, agradecer al Dr. Vicente Herrera y Dra. Vanessa Valdés, por su asesoramiento y apoyo; a la Autoridad para la 
Micro, Pequeña y Mediana Empresa, oficina provincial de Bocas del Toro; Al magíster Julio Aguilera presidente de la Cámara de Comercio, Agropecuaria e Industrial de Bocas del Toro; Al Instituto Nacional de Estadística y Censo, oficina regional de Bocas del Toro; y en especial a los propietarios $\mathrm{y} / \mathrm{o}$ administradores de las pequeñas y medianas empresas del Distrito de Changuinola por confiar en este trabajo.

\section{Referencias}

[1] G. Ekuobase y V. Olutayo, «Study of Information and Communication Technology (ICT) maturity and value: The relationship,» Egyptian Informatics Journal, vol. 17, $\mathrm{n}^{\circ} 3$, pp. 239-249, 112016.

[2] A. Mačiulytė-Šniukienè y E. Gaile-Sarkane, «Impact of information and telecommunication technologies development on labour productivity,» Procedia, social and behavioral sciences, vol. 2014, $\mathrm{n}^{\circ}$ 110, pp. 1271-1282, 2013.

[3] D. Wanyoike, E. Mukulu y A. Waititu, «ICT Attributes as Determinants of E-commerce Adoption by Formal Small Enterprises in Urban Kenya,» International Journal of Business and Social Science, vol. 3, n 23, pp. 65-74, 122012.

[4] Unión Internacional de las Telecomunicaciones, «Evaluación de las TIC para el desarrollo económico y social,» 2006. [En línea]. Available:

https://robertoigarza.files.wordpress.com/2008/11/repevaluacion-de-las-tics-para-el-desarrollo-social-resumen-uit2006.pdf. [Último acceso: 2011 2016].

[5] ITU, «Measuring the Information Society Report. Volume 2. ICT Country profiles,» Geneva, 2017.

[6] S. Rovira, P. Santoleri y G. Stumpo, «ICTs adoption in Latin American firms: the use and disuse of public policies to encourage their dissemination,» 1 2013. [En línea]. Available: https://mpra.ub.uni-

muenchen.de/65711/1/MPRA_paper_65711.pdf. [Último acceso: $5112016]$.

[7] A. Tarutè y R. Gatautis, «ICT Impact on SMEs Performance,» Procedia - Social and Behavioral Sciences, vol. 110, pp. 12181225, 12014.

[8] INEC, «Cuadro 14. Empresas en la República, por naturaleza jurídica, según nivel de ingreso y provincia: año 2009,» 2009. [En línea].

Available: https://www.contraloria.gob.pa/inec/Publicaciones/Publicacione s.aspx?ID_SUBCATEGORIA=54\&ID_PUBLICACION=510\&I D_IDIOMA=1\&ID_CATEGORIA=10. [Último acceso: 211 2016].

[9] Asamblea Legislativa, «LEY N ${ }^{\circ} 8$ De la Autoridad de la Micro, Pequeña y Mediana Empresa,» Changuinola, 200.

[10] R. Gonzalez, «Capacitación: prioridad para PYMES panameñas,» $2362015 . \quad$ [En línea]. Available:
http://www.anpanama.com/3839-Capacitacion-Prioridad-paraPYMES-panamenas.note.aspx. [Último acceso: 154 2017].

[11] M. Perez, A. Martinez, P. De Luis y M. Vela, «Las TIC en las PYMES: Estudio de resultados y factores de adopción,» 2006. [En línea]. Available: http://www.minetad.gob.es/Publicaciones/Publicacionesperiodic as/EconomiaIndustrial/RevistaEconomiaIndustrial/360/3P93105_\%20Ei\%20360-10.pdf. [Último acceso: 111 2016].

[12] L. Berrio, «PYMES crecen con el uso de herramientas tecnológicas,» $6 \quad 9$ 2016. [En línea]. Available: http://www.capital.com.pa/pymes-crecen-con-el-uso-deherramientas-tecnologicas/. [Último acceso: 1012 2016].

[13] N. Hernandez, E. De La Garza y L. Falcon, «Análisis Comparartivo de factores competitivos de las PYMES del estado de Colima, Durango y Veracruz,» Revista Global de Negocios, vol. 2, n 2, pp. 113-123, 2014.

[14] J. A. Hoyos y A. Valencia, «El papel de las TIC en el entorno organizacional de las PYMES,» Trilogía Ciencia Tecnología Sociedad, n ${ }^{\circ}$, pp. 105-122, 122012.

[15] C. E. Carlos-Ornelas, D. Ábrego Almazán, M. Aguilar , L. Aguilera, M. Fernando, M. Camarena y L. Galván, «Paradigmas Emergentes en Ciencias Administrativas y Desarrollo Regional,» 2016. [En línea]. Available: https://www.researchgate.net/profile/Monica_Sanchez_Limon/p ublication/309397837_La_calidad_de_los_servicios_publicos_b asados_en_la_percepcion_de_los_usuarios_del_Supremo_Tribu nal_de_Justicia_del_Estado_de_Tamaulipas/links/580e379e08ae 7525273d12b4.pdf\#page=60. [Último acceso: 201 2017].

[16] Fundación EOI, «Las nuevas tecnologías TIC y su impacto en la competitividad de las Pymes y en la demanda de empleo con nuevos perfiles profesionales,» 11 2003. [En línea]. Available: https://www.eoi.es/es/savia/publicaciones/20484/las-nuevastecnologias-tic-y-su-impacto-en-la-competitividad-de-laspymes-y-en-la-demanda-de-empleo-con-nuevos-perfilesprofesionales. [Último acceso: 111 2016].

[17] E. Zevallos, «Obstáculos al desarrollo de las pequeñas y medianas empresas en América Latina,» 6 2006. [En línea]. Available: http://jefas.esan.edu.pe/index.php/jefas/article/view/186. [Último acceso: 2010 2016].

[18] E. Zevallos, «Obstáculos al desarrollo de las pequeñas y medianas empresas en América Latina,» 2006.

[19] C. Ossa, «La Educación Continua un Modelo de Desarrollo,» 10 $42017 . \quad$ [En línea]. Available: https://revistaempresarial.com/educacion/diplomados-yespecializaciones/educacion-continua-modelo-desarrollo/. [Último acceso: 144 2017].

[20] R. Hernández Sampieri, C. Fernández Collado y P. Baptista Lucio, Metodología de la investigación, Sexta ed., México: McGraw Hill, 2014, p. 613.

[21] M. d. R. Silva y M. L. Brain, Validez y confiabilidad del estudio socioeconómico, México: UNAM, 2006. 